\title{
EchoGéo
}

27 | 2014

Structures et armatures urbaines

\section{Structures et armatures urbaines}

\section{Christian Vandermotten}

\section{OpenEdition}

Journals

Édition électronique

URL : https://journals.openedition.org/echogeo/13793

DOI : 10.4000/echogeo. 13793

ISSN : 1963-1197

\section{Éditeur}

Pôle de recherche pour l'organisation et la diffusion de l'information géographique (CNRS UMR 8586)

\section{Référence électronique}

Christian Vandermotten, «Structures et armatures urbaines », EchoGéo [En ligne], 27 | 2014, mis en ligne le 20 mars 2014, consulté le 10 août 2021. URL : http://journals.openedition.org/echogeo/13793 ; DOI : https://doi.org/10.4000/echogeo.13793

Ce document a été généré automatiquement le 10 août 2021.

EchoGéo est mis à disposition selon les termes de la licence Creative Commons Attribution - Pas d'Utilisation Commerciale - Pas de Modification 4.0 International (CC BY-NC-ND) 


\title{
Structures et armatures urbaines
}

\author{
Christian Vandermotten
}

1 L'éventail, du micro au macro, des articles reçus et retenus pour ce numéro d'Echogéo consacré aux structures et aux armatures urbaines incite à replacer les questions qu'ils posent dans une perspective historique, étalée sur le dernier demi-siècle. Ces questions portent sur ce dont traitent les articles, explicitement et en filigrane, mais tout autant sur ce qu'ils n'évoquent pas.

2 Quels eussent vraisemblablement été les grands thèmes traités dans un tel numéro il y a quatre ou cinq décennies, durant la période fordiste de croissance rapide, à base industrielle, de l'économie européenne?

3 Tout d'abord la question des métropoles d'équilibre et du maillage urbain : la vision normative d'une structuration des territoires par le renforcement des grandes villes régionales et par une distribution des services, hiérarchisée, pyramidale, à travers les différents niveaux de l'armature urbaine. Les objectifs étaient à la fois la déconcentration industrielle de l'île-de-France et la revitalisation/industrialisation des petites villes et des campagnes souffrant d'exode rural et de la disparition d'une agriculture employant une main-d'œuvre abondante. Ces régions rurales devaient être mises au service d'une industrie taylorisée à la recherche de bassins de recrutement, dans des temps de plein emploi.

4 Ensuite, l'analyse de la périurbanisation, en termes de croissance rapide de la population, par contiguïté et anneaux successifs à partir des limites des plus grandes agglomérations morphologiques. Périurbanisation voulue dans le chef des catégories sociales supérieures ou intermédiaires, plus contrainte par les conditions d'accès au logement pour les catégories sociales inférieures, mais chez celles-ci aussi idéologiquement valorisée, même quand elle se réalisait dans des ensembles de HLM. Il est vrai que ces derniers n'étaient pas socialement stigmatisés comme ils le sont aujourd'hui et représentaient le plus souvent un incontestable progrès en termes de qualité du logement par rapport aux conditions antérieures.

5 Enfin, la question des infrastructures, de leurs tracés et éventuellement des impacts de ceux-ci. L'analyse eût porté sur trois échelles différentes: les grands tracés autoroutiers, puis ferroviaires, structurants, entre les métropoles d'équilibre et Paris ; 
les infrastructures assurant la desserte polarisée des territoires; la desserte intraurbaine, en particulier en termes d'aménagements routiers. Ces infrastructures, impliquant des financements importants, résultaient d'impulsions et d'une planification pensées au niveau d'une imbrication étroite entre le politique et les grands corps techniques de l'État. Présentées comme des conditions indiscutables du développement économique et de la modernité, la littérature géographique les examinait d'un point de vue technocratique, se limitant à les décrire, à examiner leur contribution potentielle au développement régional, tout au plus à discuter des modalités de leur implantation.

6 Dans une certaine mesure, une telle situation et ce type d'analyse $e^{1}$ apparaissent encore dans le seul article consacré à un pays émergent, la Chine (ainsi que dans des textes proposés, mais non retenus, sur l'évolution d'armatures urbaines en Afrique). En effet cet article montre le développement industriel de l'intérieur de la province du Zhejiang, diffusant au départ des plus grandes conurbations côtières qui pour leur part sont déjà en voie de tertiarisation et dont la structuration interne s'affirme. Cette industrialisation s'accompagne d'une urbanisation galopante, s'appuie sur un réseau de villes petites et moyennes (à l'échelle chinoise) qui se renforcent relativement et «équilibrent » le territoire, assurent une "meilleure" répartition des populations et des activités économiques. Ces développements urbains vont de pair avec la mise en place de relations ferroviaires rapides. L'encadrement par ces villes de territoires densément peuplés s'accompagne d'un renforcement de leur autonomie et emprise administratives, qui s'impose aux zones rurales.

7 Qu'en est-il en revanche en Europe occidentale, du plus global au plus local, sachant que les deux niveaux d'échelle supérieurs ne sont pas abordés dans les articles proposés ici?

8 Au niveau le plus global du discours politique, la tendance est à la promotion d'un discours en faveur du polycentrisme, concept flou, polysémique, dont les échelles de pertinence ne sont le plus souvent pas précisées (Vandermotten, Halbert, Roelandts, Cornut, 2008). Ce discours s'appuie sur le postulat, non empiriquement vérifié, que plus de polycentrisme assurerait un meilleur équilibre et un meilleur développement à l'ensemble du territoire européen et fonderait un développement plus écologique et durable. Tenu à l'échelle européenne, il recueille le consensus en sous-entendant que les politiques ne délaisseront aucune portion du territoire de l'Union, alors même que la tendance penche moins vers une cohésion accrue qu'en faveur de plus de compétitivité. On a montré que ceci conduit au contraire à un renforcement de l'espace central européen, aux dépens des zones périphériques (Lennert \& Robert, 2007). Transposé aux échelles nationales, le discours en faveur du polycentrisme satisfait les régions et les villes, qui y trouvent chacune des raisons d'espérer. Elles appuient leurs ambitions sur des benchmarkings aux méthodologies et aux conclusions stéréotypées. En résultent des politiques régionales et locales qui de fait dégagent des effets d'aubaine pour les investisseurs, sans qu'elles ne modifient réellement leurs logiques de comportement spatial. Tout cela dédouane les États de leur désengagement en matière d'intervention sur les territoires.

9 Au niveau hiérarchique le plus élevé des réalités urbaines, la métropolisation se développe dans le cadre de la globalisation de l'économie mondiale et de la tertiarisation de l'économie des pays du centre. Nous parlons ici de la réelle métropolisation, pas de celle dont se revendiquent aujourd'hui des villes de tout niveau hiérarchique, parfois 
parmi celles dont les structures sont les plus éloignées des caractéristiques de la métropolisation. En effet, ce concept signifie insertion de la (très) grande ville en tant que nœud d'accessibilité internationale ; comme nœud dans les réseaux internationaux des services financiers, juridiques, de marketing, techniques, de commandement de l'économie transnationale; comme lieu de production ou en tout cas de contrôle de la production technologique; comme lieu culturel majeur. La description de ces réseaux et de leurs nœuds, leur fonctionnement, sont bien documentés, par exemple par les travaux du $\mathrm{GaWC}^{2}$, dirigés par P.J. Taylor et J.V. Beaverstock, ou, dans le monde francophone, de plusieurs auteurs, dont C. Rozenblat. À la suite de Florida (2005), les réussites économiques de cette métropolisation valorisent et sont valorisées par une littérature sur les industries créatives qui s'y concentrent et sur la rénovation métropolitaine à travers la gentrification d'une part, les grands projets emblématiques, les "flagships", de l'autre. Cette littérature dominante se préoccupe peu des conséquences sociales négatives de ces succès économiques. Concentrant dans ces métropoles cosmopolites les cadres du plus haut niveau, ils y exaspèrent les dichotomies sociales, y enflamment les prix de l'immobilier et y génèrent une croissance qui fournit trop peu d'emplois pour les populations les moins qualifiées. Il en résulte une croissance qui s'accompagne souvent de niveaux élevés de chômage, du moins dans les quartiers les moins privilégiés de ces vastes ensembles métropolitains. Il existe aussi heureusement une littérature critique sur ces thèmes.

Toutefois, si dans les périphéries, même la périphérie européenne, les métropoles, ou du moins les plus grandes villes, enregistrent les performances économiques les plus favorables, dans les parties les plus denses et les plus développées de l'Europe, des régions encadrées par des villes moyennes peuvent aussi enregistrer de bonnes performances, du moins quand leurs structures sont favorables à une croissance postindustrielle et qu'elles peuvent valoriser des économies de niche dans des secteurs porteurs, voire des atouts culturels et patrimoniaux (David, Peeters, Van Hamme \& Vandermotten, 2013). C'est qu'en Europe du nord-ouest, les plus grandes aires métropolitaines subissent des déséconomies d'échelle, alors que l'accessibilité et la qualité des infrastructures, des équipements et de la main-d'œuvre peuvent être d'un excellent niveau dans des villes moyennes, qui ne sont d'ailleurs souvent pas très éloignées des plus grandes métropoles. D'où l'intérêt porté aux villes régionales et au développement de systèmes urbains régionaux. L'article de Gingembre et Baude sur "Les mobilités domicile - travail dans les réseaux d'agglomération" montre une autonomisation de ces systèmes, à l'intérieur desquels se développe une circulation de cadres; il conviendrait de le compléter par une analyse liant les performances économiques (et sociales) de ces systèmes régionaux à leurs structures et éventuellement aux réalités de leur gouvernance. En tout cas, l'article montre bien que si ces systèmes en émergence ont pu favoriser les mobilités de la main-d'œuvre et donc les potentialités d'emploi, ce sont avant tout les cadres qui en bénéficient.

11 À l'échelle de chaque agglomération morphologique, la périurbanisation ne répond plus à l'image relativement simple qui la caractérisait précédemment: le départ vers les franges extérieures de la banlieue de jeunes couples avec enfants, en quête de logement à un prix plus accessible, dans un environnement jugé plus agréable, au prix de déplacements de travail quotidiens centripètes vers les parties centrales des agglomérations. Aujourd'hui, comme Berger, Aragau et Rougé le montrent dans le cas de l'ouest francilien, mais le phénomène peut s'observer partout en Europe occidentale, les aires périurbaines, du moins autour des plus grandes villes, ont développé une 
structuration propre, avec une multiplication de centres d'emploi et de services. Leurs populations se sont diversifiées dans leurs structures par âge et par statut familial ou professionnel. Elles se sont aussi stabilisées et sont moins souvent des primo-émigrants issus de la ville-centre. Certaines parties du périurbain, en particulier celles les plus proches des villes-centres, ont vieilli, et certaines de leurs communes connaissent une baisse de population, non seulement du fait de leur vieillissement, mais aussi par bilan migratoire devenu négatif. Le périurbain s'est étendu, souvent de manière non contigüe, pour se transformer en rurbain, et tend à reporter les moins nantis vers ses marges les plus éloignées. Mais pourtant les distances moyennes parcourues par les périurbains pour leurs déplacements domicile - travail n'augmentent plus que très peu, du fait de l'émergence de noyaux secondaires.

12 Reste enfin l'échelle intra-urbaine, traitée par Demailly à travers la question des jardins partagés. Certes, on pourrait parler ici d'une micro-géographie, à la limite du dérisoire. Mais, d'une part, elle est exemplaire d'une certaine géographie post-moderne, largement pratiquée par les Anglo-saxons, alliant qualitatif et démarche proche de celle des sociologues urbains, privilégiant la compréhension, voire simplement la description, des comportements dans la ville à l'analyse des structures spatiales. Surtout, d'autre part, elle est révélatrice des évolutions de la gouvernance.

13 À nouveau, la comparaison avec la situation qui prévalait durant la période fordiste s'impose : l'État pour les orientations générales et l'implémentation des grands projets, les pouvoirs locaux pour les autres, étaient sans conteste à la manœuvre, dans une démarche top-down à laquelle commençait à peine à s'opposer en fin de période, très ponctuellement, l'une ou l'autre contestation citoyenne, en général vite balayée.

Aujourd'hui, l'État tend à se désengager, ou se limite à dessiner de grandes orientations stratégiques. Il conserve toutefois une plus grande emprise en France que dans d'autres pays ouest-européens aux structures beaucoup plus régionalisées ou fédérales, où il n'a parfois plus, comme en Belgique, aucune compétence en la matière.

Une partie plus ou moins importante des responsabilités en matière de développement territorial et urbain est donc reportée sur les Régions et les villes, sans toujours qu'elles ne disposent des moyens financiers adéquats, ce qui pousse vers différentes formes de partenariat public - privé, voire de désengagement complet en faveur du privé, justifiées au nom d'une soi-disant plus grande efficacité ${ }^{3}$. Toutefois, les cadres politiques dans lesquels cette décentralisation est développée ne correspondent plus toujours aux réalités contemporaines des fonctionnements urbains et régionaux. Si l'article sur les mobilités dans les réseaux d'agglomération montrait que dans la plupart des cas ceux-ci s'inscrivent en France à l'intérieur des cadres régionaux, il existe toutefois des exceptions qui interpellent, par exemple quant au maintien de deux Régions en Normandie ou au contraire à la fonctionnalité d'une Région Rhône-Alpes unique réunissant le système urbain lyonnais et ceux de l'espace franco-genevois et du sillon alpin, qui apparaissent indépendants l'un de l'autre. Mais toute modification des cadres territoriaux est difficile : elle doit vaincre les inerties des instances politiques installées et aussi les égoïsmes. On l'a vu dans le rejet par les populations néerlandaises des banlieues plus aisées d'accepter la création de provinces métropolitaines qui auraient pu mutualiser les charges des agglomérations centrales et les ressources des habitants de ces banlieues aisées. En Belgique, le refus de l'extension des limites de la Région-Capitale ou même les freins à la mise en place effective d'une vaste région métropolitaine réunissant la capitale et sa périphérie et dont les compétences seraient 
pourtant très limitées ne relèvent pas seulement de facteurs linguistiques, mais tiennent aussi à la volonté des pouvoirs locaux périphériques de ne pas avoir à subir les charges et les coûts d'une centralité, dont profite pourtant tous les jours leurs habitants-navetteurs.

Dans le contexte général de promotion de l'action du secteur privé et de compétition entre les villes sous-tendue par les références de l'économie globalisée, coincés aussi par la faiblesse de leurs moyens financiers, les pouvoirs locaux sacrifient aux logiques dominantes: l'action des pouvoirs publics doit favoriser la gentrification, une esthétique de qualité, l'attractivité culturelle, celle des créatifs. Si on comprend bien les évolutions que les pouvoirs locaux de droite attendent de telles évolutions, l'article de Lebeau sur "Une banlieue "créative" dans le Grand Paris?" montre que les municipalités de gauche y sacrifient aussi, même si elles tentent de donner un vernis plus « démocratique » à ces options. L'article indique bien qu'il est illusoire d'espérer développer une "cité créative", attractive au sens des critères de l'économie dominante et en même temps socialement intégratrice, au départ des formes les plus révoltées et les plus alternatives de la culture populaire. Hors de France, l'exemple bruxellois montre lui aussi que les municipalistes socialistes sont d'autant plus enclins à vouloir "éradiquer" la pauvreté urbaine à travers un encouragement de la gentrification, à travers des politiques d'esthétisation des espaces urbains, qu'ils gèrent des municipalités pauvres et sont donc à la recherche de ressources fiscales accrues, voire de réductions des charges qu'ils doivent supporter du fait même de la structure sociale de leurs communes.

17 Cette gentrification, cette volonté d'accueillir les classes moyennes, les « créatifs », les populations bien éduquées, impose évidemment aux autorités locales de leur laisser des espaces de participation. Mais l'article sur «Les jardins partagés franciliens » montre bien les limites de celle-ci : les décisions restent en fin de compte sous le contrôle des autorités politiques, même pour les initiatives qui apparaissent comme les plus spontanées.

\section{BIBLIOGRAPHIE}

David Q., Peeters D., Van Hamme G. \& Vandermotten C., 2013. Is bigger better? Economic performances of European cities, 1960-2009. Cities, 35, p. 237-254.

Florida R., 2005. Cities and the Creative Class. Routledge.

Lennert M. \& Robert J. (dir.), 2007. Scenarios for the territorial future of Europe. ESPON, Program 3.2 et ibid. (2007), Territorial futures. Spatial scenarios for Europe. ESPON, Program 3.2.

Vandermotten C., Halbert L., Roelandts M., Cornut P., 2008. European Planning and the Polycentric Consensus : Wishful Thinking? Regional Studies, 42, 8, p. 1205-1217. 


\section{NOTES}

1. Y compris, implicitement, des considérations normatives : « les petites villes équilibrent peu à peu l'armature urbaine ", " un chapelet éclectique de villes qui aujourd'hui atteignent déjà leur taille critique ", "une meilleure répartition des populations et des activités économiques sur le territoire ", etc.

2. www.lboro.ac.uk/gawc.

3. Avec la variante britannique, où l'État tend à priver de leurs compétences les pouvoirs locaux, faibles mais qu'il ne contrôle pas toujours, au profit d'agences nationales ou ad-hoc, qui délèguent au privé ou à des partenariats public - privé.

\section{INDEX}

Thèmes : Sur le Champ - Sur le Terrain

\section{AUTEUR}

\section{CHRISTIAN VANDERMOTTEN}

Christian Vandermotten, cvdmotte@ulb.ac.be, est Professeur ordinaire émérite à l'Université libre de Bruxelles, Belgique. Il a publié récemment :

- Vandermotten C., Marissal P. et Van Hamme G., 2014. La production des espaces économiques. Bruxelles, Editions de l'Université de Bruxelles, 2 vol., 4ème éd.

- Vandermotten C., 2013. Pour un développement économique régional inclusif du social. In Eric Corijn et al., Où va Bruxelles. Visions pour la capitale belge et européenne, Bruxelles, VUBPress, p. 81-105.

- Vandermotten C., 2012. Cotation des revues de géographie, impérialisme scientifique anglosaxon et culture de l'excellence marchandisée. Belgeo, 2012, 1-2, 16 p., http://belgeo.revues.org/ 7131. 